\title{
ATG5 and ATG7 induced autophagy interplays with UPR via PERK signaling
}

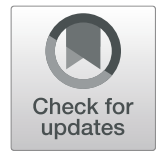

Wei Zheng ${ }^{\dagger}$, Weiwei Xie ${ }^{\dagger}$, Danyang Yin ${ }^{\dagger}$, Rui Luo, Min Liu and Fengjin Guo

\begin{abstract}
Background: Autophagy and ER stress are involved in maintaining some well-orchestrated mechanisms aimed at either restoring cellular homeostasis or performing cell death. Autophagy is a well-defined process which governs overall cellular stress outcomes. Selective degradation of the ER mediated by autophagy occurs through a specific type of autophagy called ER-phagy, which ensures ER protein homeostasis.

Methods: Immunoblotting and RT-PCR were used to evaluate the expression of ATG5 and ATG7 in chondrocyte. Western blotting, Flow cytometry,immunofluorescence cell staining and confocal microscope were used to examine the effect of ATG5 and ATG7 on autophagy, ER stress, cell apoptosis and cell proliferation. Transmission electron microscope and confocal microscope were performed to visualize the autophagy flux and autolysosome formation. The role of ATG5 and ATG7 overexpression on the PERK pathway inhibitor were detected by immunoblotting and treatment with inhibitors.
\end{abstract}

Results: In current study, we demonstrated that Tm-induced ER stress can activate autophagy while Rapamycininduced autophagy can inhibit ER stress in chondrocyte. Autophagy related protein ATG5 or ATG7 can promote autophagy and inhibit ER stress individually, and their combined effect can further improve the autophagy enhancement and the ER stress repression. Moreover, ATG5, ATG7 and ATG5 + ATG7 lead cells into more S phase, increase the number of S phase and inhibit apoptosis as well. ATG5, ATG7 and ATG5 + ATG7 regulate autophagy, ER stress, apoptosis and cell cycle through PERK signaling, a vital UPR branch pathway.

Conclusions: ATG5 and ATG7 connect autophagy with ER stress through PERK signaling. The protective effect of ATG5/7 overexpression on chondrocyte survival relys on PERK signaling. The effect of siPERK and siNrf2 on the cytoprotective effect of ATG5/7 are of synergism, while the effect of siPERK and siATF4 are of antagonism. PERK signal may be the pivot for autophagy, ER homeostasis and ER-phagy in chondrocyte.

Keywords: ATG5, ATG7, Autophagy, ER stress, ER-phagy, Apoptosis

\section{Background}

The endoplasmic reticulum (ER) is an elaborate cellular organelle essential for cell function and survival. Autophagy, ER stress and apoptosis are closely connected with ER. It's well known that autophagy in mammalian systems occurs under basal conditions and can be stimulated by stresses like hypoxia, starvation, rapamycin etc. Autophagy can prevent cells from many kinds of stress and was beneficial for cell survival.

In the process of autophagy, the damaged or dysfunctional organelles and macromolecules are encapsulated

\footnotetext{
* Correspondence: guo.fengjin@gmail.com; guo.fengjin@cqmu.edu.cn tWei Zheng, Weiwei Xie and Danyang Yin contributed equally to this work. Department of Cell Biology and Genetics, Core Facility of Development
} Biology, Chongqing Medical University, Chongqing 400016, China in the double membrane structure called autophagosome which will then degrade the macromolecule components after fusing with the lysosomes to form autolysosomes to maintain homeostasis of the cells [1-3]. Cell death will happen when autophagy is inhibited, implying autophagy as a cytoprotective mechanism $[4,5]$. There are two ubiquitin-like conjugatin systems necessary for the phagophore membrane elongation, including ATG12-ATG5ATG16L1 autophagosomal precursor formation [6-8] and LC3-I/LC3-II production, which is involved in fusing autophagosome with lysosome to form autolysosomes [9-11]. All is known that autophagy function and morphology are intimately associated to ER, which is necessary for the cell survival under normal condition. The ER stress

(c) The Author(s). 2019 Open Access This article is distributed under the terms of the Creative Commons Attribution 4.0 International License (http://creativecommons.org/licenses/by/4.0/), which permits unrestricted use, distribution, and 
will be stimulated once beyond the function of the ER [12-14], and the unfolded protein response (UPR) will be activated when some endogenous or exogenous factors influence the homeostasis of ER. ER-phagy exists after selective degradation of the ER by autophagy,and play a key role in the physiology of secretory cells in vivo. ER stress and UPR directly engage and modulate general autophagic flux and direct ER-phagy. Smith et al. identify ER membrane protein CCPG1, as an ER-phagy receptor that interacts with autophagy-related components LC3, GABARAPs and FIP200, maintains ER homeostasis during both physiological and stress conditions [15-17].

Many studies reported that a variety of physical and chemical factors can turn on ER stress and influence cell survival in chondrocyte differentiation, chondrogenesis and endochondral ossification [18-20]. ER stress-induced cell apoptosis will be switched on when stress continues to occur or the cell is unable to accommodate ER stress [21-23]. ER stressors, like tunicamycin, thapsigargin, or DTT, stimulate the autophagosomes formation [24]. The activation of autophagy under ER stress may have a cytoprotective effect and promote cell survival [25-27]. ATG5 and ATG7, as two important autophagy related proteins, increased antophagy and reduced the damaged organelles or degraded macromolecules which accumulated in chondrocytes of cartilage degeneration, then maintained the homeostasis of chondrocyte and were conducive to cell survival [28-30]. However, when and how to modulate autophagy during ER stress is not entirely clear,the direct correlation between these two processes remains unknown. This study aim to clarify the effect of ATG5 and ATG7 on how to regulate ER stress, autophagy and cell survival. Specifically, the data presented herein elucidate the relationship between autophagy, ER stress and ER-phagy. ATG5 and ATG7, as two conventional autophagy-related genes, are involved in ER turnover through PERK signaling. It is of significant interest to clarify the reason behind treatment with autophagy inducer is beneficial to the removel of cytosolic aggregates.

\section{Methods}

\section{Adenoviruses and plasmids}

To generate ATG5 and ATG7 overexpression adenovirus, the cDNA of ATG5 and ATG7 genes were cloned into the pAdTrack-CMV and recombinated to adenovirus according to the instruction (primers: forward, $5^{\prime}$-GTCA GATCCGCTAGAGATCT GCTTACTAAGTTTGGCTT TGGTT-3' and reverse, 5'-GATATCTTATCTAGAAGC TTAAGGGTGACATGCTCTGATAAAT-3' for ATG5; forward, 5'-GTC AGATCCGCTAGAGATCTAAATAAT GGCGGCAGCTACGG-3' and reverse, 5'-GATATCTT ATCTAGAAGCTTGGGCCATCTCAGATGGTCTCAT
C-3' for ATG7) [30]. Futhermore, the cDNA of ATG7 (forward, 5' -GTTTAAACGGGCCCTCTA GAAAATAAT GGCGGCAGCTACGG-3' and reverse, 5'-TGGAATTCT GCAGATA TCGGGCCATCTCAGATGGTCTCATC-3') was cloned into pcDNA3.1(-) and pcDNA3.1(-)-ATG5 was presented by Dr. Chuanju Liu of New York University. All of the constructs were verified by endonuclease digestion and nucleic acid sequencing.

\section{Cell culture}

C28I2 cells (a gift from Dr. Chuanju Liu, New York University School of Medicine, New York, NY, USA) were cultivated in DMEM (Gibco, Grand Island, NY, USA) medium supplemented with $10 \%$ fetal bovine serum in $10 \%$ fetal bovine serum supplemented DMEM (Gibco, Grand Island, NY, USA), and incubated at $37^{\circ} \mathrm{C}$ in a humidified atmosphere of $5 \% \mathrm{CO} 2$. Next, the cells were briefly trypsinized and resuspended into the $60 \mathrm{~mm}$ cell culture dish (Biologix, USA), then incubated for $12 \mathrm{~h}$ under standard conditions before treatment. To confirm the effects of ATG5, ATG7, siPERK and siNrf2 on chondrocyte. C28I2 cells were infected with Ad-ATG5 $(\mathrm{MOI}=80)$, Ad-ATG7 $(\mathrm{MOI}=100)$, siPERK $(50 \mathrm{nmol})$ or siNrf2 $(50 \mathrm{nmol})$ and Ad-GFP prior to culture $24 \mathrm{~h}$ $[31,32]$. pcDNA3.1(-)-ATG5 and pcDNA3.1(-)-ATG7 were transfected into cells for $24 \mathrm{~h}$ by liposome 8000 (Invitrogen) according to the manufacturer's protocol.

\section{RNA extraction and reverse transcription (RT)-PCR}

RNeasy Mini Kit (BioTeke, Chinese) and PrimeScript RT reagent Kit (TAKARA, USA) were used to extract total RNA and reverse transcription respectively based on the instructions. The specific sequence primers were designed as follows: forward, 5 -AAGCAACTCTGGAT GGGATT-3' and reverse, 5'-GCAGCCACAGGACGA AAC-3' for ATG5; forward, 5'-CAGTCCGTTGAA GTCCTC-3' and reverse, 5'-TCAGTGTCCTAGCC ACATTAC-3' for ATG7; forward, 5'-AGGTCGGTG TGAACGGATTTG-3' and reverse, 5'-GGGGTCGTT GATGGC AACA-3' for GAPDH. The targeted PCR amplification products were verified by gel-purified bands (Qiagen). The quantitation of RNA was analysed by the BIO-RAD CFX Connect Real-Time PCR system (BIO-RAD). GAPDH was employed as an internal control. And annealing temperatures for these primers were $55^{\circ} \mathrm{C}$ except ATG5 which was $52^{\circ} \mathrm{C}$. Data were analyzed by the Relative Quantification $\left(2^{-\Delta \Delta \mathrm{Ct}}\right)$ method.

\section{Western blotting}

Total proteins were extracted by RIPA lysis buffer which mixed with proteinase inhibitor (PMSF). The proteins were next separated by $12 \%$ SDS-PAGE and transfered to the PVDF membrane (Millipore). Then blocking for 
$2 \mathrm{~h}$ in $5 \%$ non-fat dry milk which was dissolved with $1 \times$ Tris-buffered saline and incubated with the corresponding antibody [ATG5 (NB110-53818; NOVUS 1:500); ATG7 (MAB6608; R\&D 1:500); LC3 (NB1002220; NOVUS 1:1000); SQSTM1/p62 (ab56416; abcam 1:1000); Caspase-3 (\#9662; CST 1:1000); Caspase-12 (ab62484; abcam 1:1000); PERK (sc-13,073; Santa Cruz 1:200); p-PERK (sc-32,577; Santa Cruz 1:200); Nrf2 (sc-722; Santa Cruz 1:500); XBP1 (sc-7160; Santa Cruz 1:200); ATF4 (\#11815; CST 1:1000); IRE1 (NB100-2323; NOVUS 1:1000); $\beta$-actin (B1033; Biodragon 1:8000)] at $4{ }^{\circ} \mathrm{C}$ overnight. The next day, the HRP-conjugated goat anti-mouse or anti-rabbit IgG was added to incubate for $2 \mathrm{~h}$ after washing for thrice. At last, an enhanced chemiluminescence reagent (Beyo ECL Plus, Beyotime) was used to visualized the band.

\section{Flow cytometry}

On basis of references [33, 34], when C28I2 cells were incubated with the adenoviruses or controls for $24 \mathrm{~h}$, the cells were washed, trypsinized and resuspended with $1 \times$ PBS for FCM assay or fixed the cells with $75 \%$ alcohol for determing the distribution of cell cycle. These experiments were conducted thrice.

\section{Immunofluorescence cell staining and confocal microscope} Cells were planted on coverslips and infected with the adenoviruses or transfected with the plasmids for $24 \mathrm{~h}$. Then washing for twice with $1 \times$ PBS and fixed the cells with $4 \%$ paraformaldehyde for $30 \mathrm{~min}$. Next permeabilized with Triton X-100 (0.1\% in PBS) for 20 min and blocked with 5\% BSA for $1 \mathrm{~h}$. Next incubating the cells with LC3 (1:200) and LAMP1 (1:200) at $4{ }^{\circ} \mathrm{C}$ overnight. The next day, the cells were incubated with HRP-conjugated goat anti-rabbit IgG (1:300, abbkine) for $1 \mathrm{~h}$ darkly at room temperature after washing with $1 \times$ PBS thrice. Then the 4-6-diamidino-2-phenylindole (DAPI) was added for $15 \mathrm{~min}$. Finally, using a confocal microscope (Nikon) to collect the corresponding images.

\section{Transmission electron microscope}

After incubated with the adenoviruses or controls for $24 \mathrm{~h}$, the cells were washed, trypsinized and resuspended.Then fixed the cells with glutaraldehyde for transmission electron microscope analysis to detect autophagosomes. Transmission electron microscope (JEM-1400PLUS) was used to collect the corresponding images.

\section{Statistical analysis}

Date were analyzed by GraphPad Prism 5 software and student's t-test for single comparisons. These data come from at least 3 independent experiments and expressed as means \pm SD. Moreover, ${ }^{*} P<0.05,{ }^{* *} P<0.01,{ }^{* * *} P<0.001$ were considered statistically significant.

\section{Results}

ER stress interplays with autophagy in human chondrocyte It is well known that when UPR is triggered in ER stress, the activation of PERK signaling pathway is initiated upon its dimerization and autophosphorylation [20-22]. Tunicamycin (Tm), as a typical ER stress inducer, can obviously elicit the ER stress, such as accumulation of PERK(125KD), phosphorylated PERK (125KD), nuclear transcription factor Nrf2 (68KD), IRE1 (110KD) and $\mathrm{XBP} 1 \mathrm{~s} / \mathrm{XBP} 1 \mathrm{u}(40 \mathrm{KD} / 29 \mathrm{KD})[35,36]$. We detected the expression of autophagy related proteins under ER stress induced by $\mathrm{Tm}$. The result showed that the ER stress-associated molecules, were activated in Tm-treated cells (Fig. 1a, c), and the expression of autophagy related proteins, including ATG5 (33KD), ATG5-ATG12 (56KD), ATG7 (78KD), LC3-I/LC3-II $(16 \mathrm{KD} / 18 \mathrm{KD})$ were also increased, the expression of P62 (62KD) was reduced after Tm treatment (Fig. 1a, b, c and d). Furthermore, we detected the expression of autophagy related proteins during ER stress inhibited by siPERK and siNrf2, the specific siRNA approach. The inhibit rate of siPERK1, SiPERK2 and siPERK3 are 78, 69 and $45 \%$ respectively. The inhibit rate of siNrf2-1, siNrf2-2 and siNrf2-3 are 65,58 and $21 \%$ respectively. The inhibit rate of siATF4-1, siATF4-2 are 38 and 56\% respectively (Fig. 1e, f).

The result showed that the expression of ATG5, ATG7, LC3-I/LC3-II were obviously reduced, and the expression of P62 was increased after siPERK and siNrf2 treatment. Knockdown expression of PERK and Nrf2 can inhibit autophagy activation(Fig. 1g, h). We then detected ER stress associated proteins expression after rapamycin (RAPA), a typical autophagy inducer, treatment, and it is showed that PERK, p-PERK and Nrf2 were depressed in the RAPA-treated chondrocyte (Fig. 1i and j).These results suggested that autophagy affected ER stress in human chondrocyte, and vice versa.

\section{Overexpression of ATG5 or ATG7 enhance autophagy and inhibit ER stress in chondrocyte}

To detect the effect of ER stress by ATG5 and ATG7, two critical autophagy related proteins, Ad-ATG5 and Ad-ATG7 adenoviruses vectors were constructed and identified with endonuclease digesting and DNA sequencing, respectively. The results showed that the construction of plasmids were correct (Fig. 1s, Additional file 1). Then the C28I2 chondrocytes infected with Ad-ATG5 or Ad-ATG7 were identified by RT-PCR,Q-PCR and Western blot. The level of ATG5 and ATG7 mRNA were obviously increased in the Ad-ATG5 and Ad-ATG7 infected cells, comparing with their controls, Ad-GFP as a control (Fig. 2a, b). And the protein levels were also significantly enhanced in the Ad-ATG5 and Ad-ATG7 infected 




cells, comparing with the other two control cells, respectively (Fig. 2c, d). The results illustrated that the construction and expression of Ad-ATG5 and Ad-ATG7 were correct.

We next to examine the expression of autophagy related protein, including LC3, P62, and ER stress associated protein, such as PERK, p-PERK, Nrf2, in the
Ad-ATG5 and Ad-ATG7 infected chondrocytes. The result showed that LC3-I/LC3-II were increased and P62 were reduced in the Ad-ATG5 and Ad-ATG7 infected chondrocytes comparing with the controls. Furthermore, the expression of PERK、p-PERK and Nrf2 were decreased in the Ad-ATG5 and Ad-ATG7 infected chondrocytes comparing with the controls (Fig. 2e and f). 


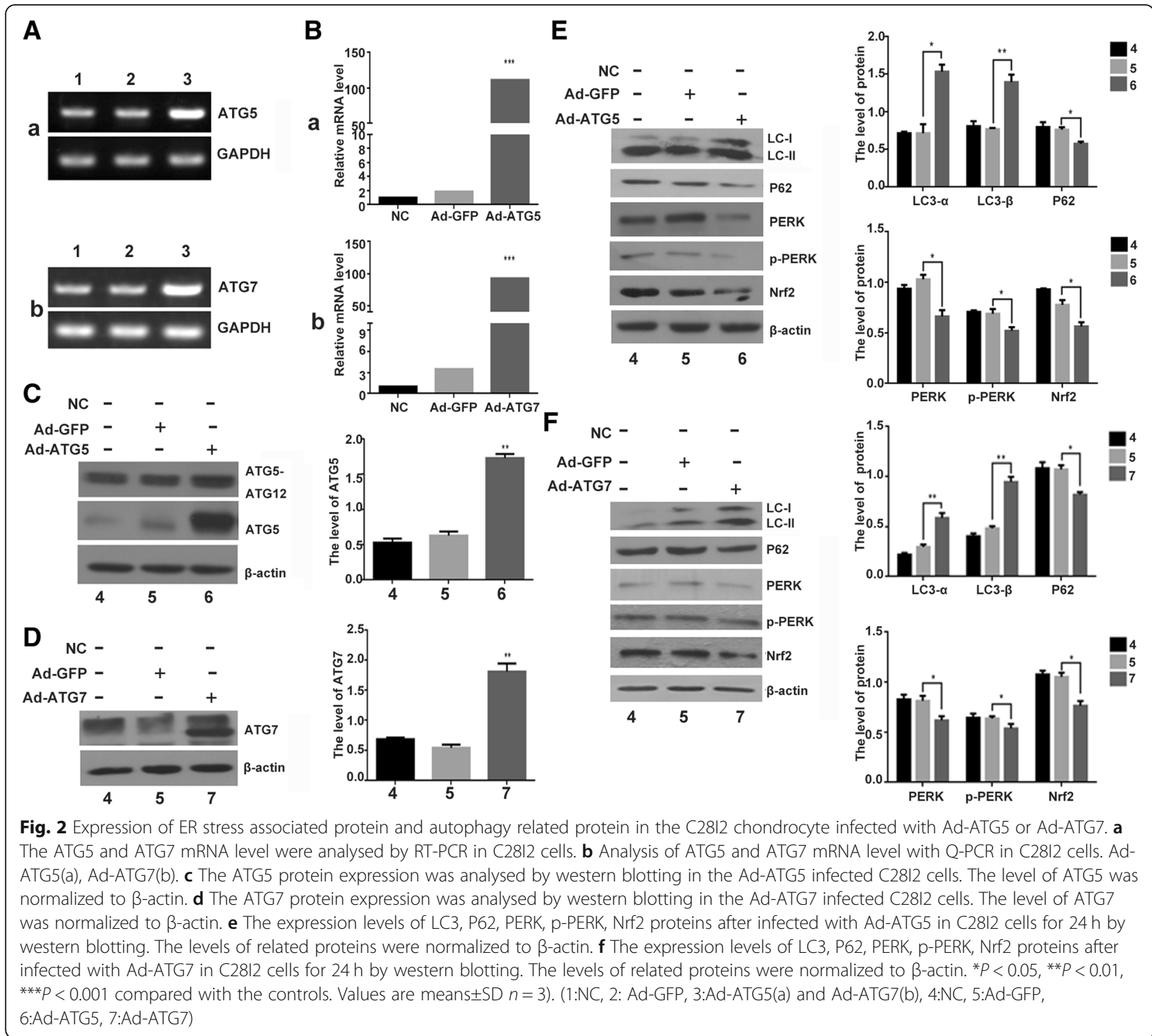

Taken together, overexpression of ATG5 or ATG7 enhance autophagy and inhibit ER stress in chondrocyte.

\section{The effect of ATG5 and ATG7 on autophagy and ER stress} in chondrocyte

Next, we examined how the ATG5 and ATG7 influence on autophagy and ER stress. As revealed in Fig. 3a and b, The level of autophagy proteins, ATG5, ATG7, ATG5-ATG12 and LC3-I/LC3-II were significantly increased after infected with Ad-ATG5, Ad-ATG7, Ad-ATG5 + Ad-ATG7 in chondrocyte, and ATG5 + ATG7 improved this enhancement furtherly. In addition, we detected the combined effect of ATG5 and ATG7 on the expression of PERK, p-PERK and $\mathrm{Nrf2}$, three kinds of ER stress related proteins. The expression of PERK, p-PERK and Nrf2 were obviously reduced after infected with Ad-ATG5, Ad-ATG7, Ad-ATG5 + Ad-ATG7 comparing with the control, and the combined ATG5 and ATG7 aggravated this repression effect. Rapamycin used as a positive control (Fig. 3e and f).

The immunofluorescence images showed that not only Ad-ATG5 or Ad-ATG7 enhanced the LC3 expression respectively, but also their combined effect significantly increased the LC3 expression comparing with their individual effect (Fig. 3c, d). Taken together, the individual effect of ATG5 and ATG7 increased autophagy and reduced ER stress, the combined of them could obviously improve the effect of autophagy enhancement and ER stress inhibition. The augment of autophagy-related protein ATG5 and ATG7 may partially abolish ER stress activation. 


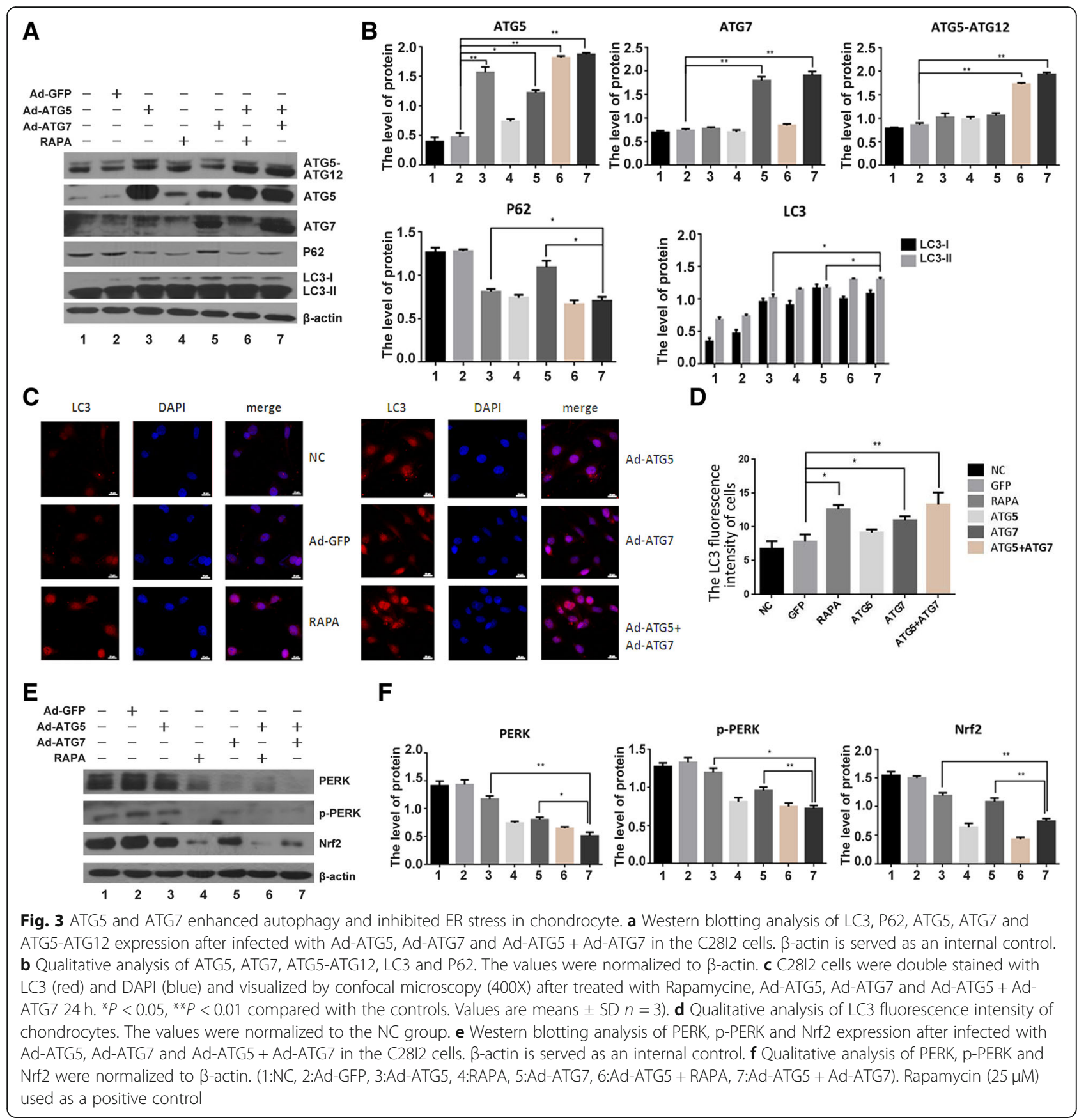

The effect of ATG5 and ATG7 on autophagy flux and autolysosome formation

It is known that lysosome-associated membrane proteins 1 and 2 (LAMP-1 and LAMP-2), as the major protein components of the lysosomal membrane, are delivered to phagosomes during autophagy process. Microtubule-associated protein $1 \alpha / \beta$-light chain 3 (LC3) is a kind of soluble protein, which is recruited to autophagosomal membranes during autophagy process. Autophagosomes fuse with lysosomes to form autolysosomes, and intra-autophagosomal components are degraded by lysosomal hydrolases [37-39].

In order to assay for autophagic flux, we detected whether autophagosome fused with lysosome and formed autolysosomes with red fluorescent-tagged LC3 and green fluorescent-tagged LAMP1. Briefly, the C28I2 cells were treated with Rapamycin $(25 \mu \mathrm{M}), \quad$ pcDNA3.1(-), pcDNA3.1(-)-ATG5, pcDNA3.1(-)-ATG7 and pcDNA3.1(-)- ATG5 + pcDNA3.1(-)-ATG7, Bafilomycin A1 $(0.4 \mu \mathrm{M})$, 
Bafilomycin $\quad$ A1 + pcDNA3.1 (-)-ATG5 + pcDNA3.1 (-)-ATG7, respectively. Then incubated with anti-LC3 antibody, anti-LAMP1 antibody and DAPI respectively. Rapamycin, a basic of autophagy inducer, can improve autophagic flux formation. Bafilomycin A1, a basic lysosomal inhibitor, can prevent the fusion of autophagosome and lysosome. The result showed that the individual treatment of ATG5 or ATG7 can increase the autolysosome formation comparing with the control. Meanwhile, the combined effect of pcDNA3.1(-)-ATG5 + pcDNA3.1(-)-ATG7 can significantly improve this enhancement effect and increase the autophagy flux furtherly, however, the augment effect of pcDNA3.1 (-)-ATG5 + pcDNA3.1(-)-ATG7 can be blocked after Bafilomycin A1 treatment (Fig. 4a, c).
Next, from the transmission electron microscope (TEM) images, the autophagosome and the autolysosome were dramaticlly increased in the Ad-ATG5 + Ad-ATG7 group compared with that of the Ad-GFP control and the individual treatment of ATG5 or ATG7. Rapamycin used as a positive control (Fig. $4 \mathrm{~b}, \mathrm{~d}$ ). These results indicated that the individual ATG5 or ATG7 could increase autophagy flux and ATG5 + ATG7 can clearly enhance this effect and augment the autolysosome formation furtherly in chondrocytes.

The effect of ATG5 and ATG7 on cell apoptosis and cell proliferation in chondrocyte

Next, we sought to determine the effects of Ad-ATG5, Ad-ATG7 and Ad-ATG5 + Ad-ATG7 on

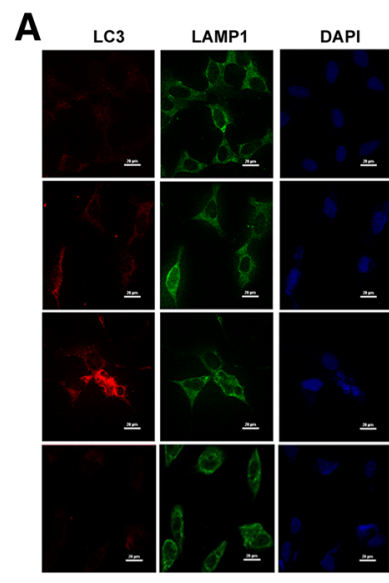

B

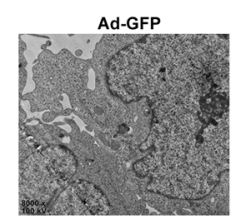

Ad-ATG5

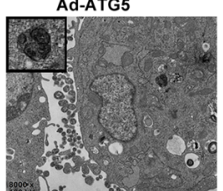

Ad-ATG5+Ad-ATG7
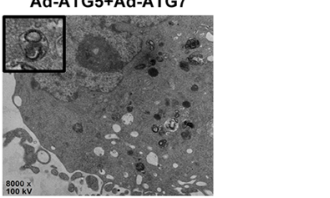

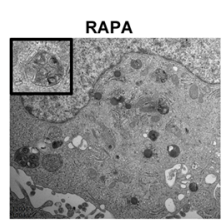

Ad-ATG7
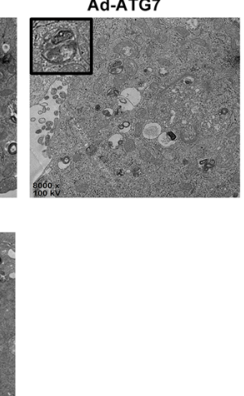

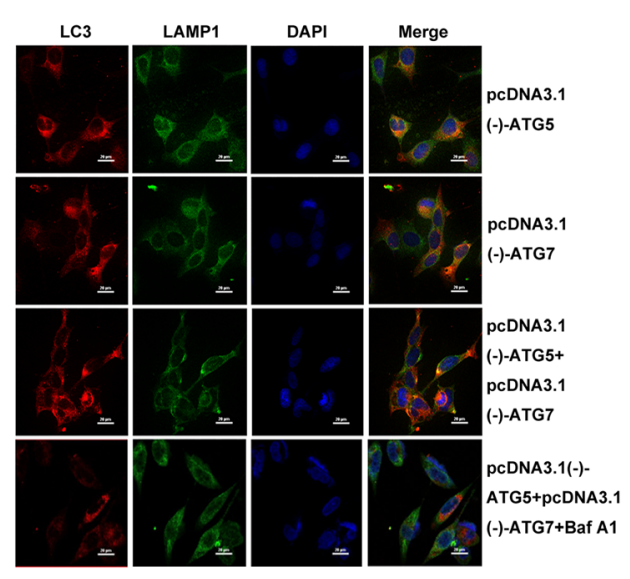

C

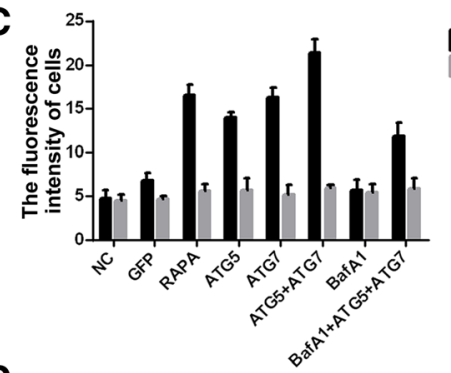

D

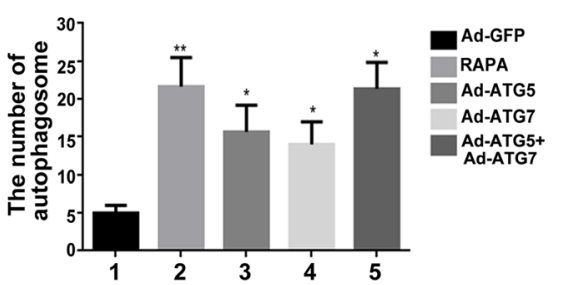

Fig. 4 Determination the effect of ATG5 and ATG7 on autophagy flux. a The C28I2 cells were treated with Rapamycin (25 $\mu M$ ), pcDNA3.1(-),

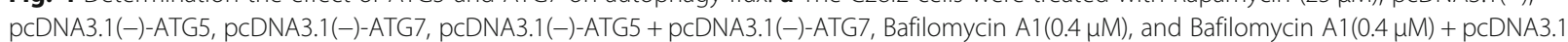
(-)-ATG5 + pcDNA3.1(-)-ATG7, then immediately stained with the anti-LC3 antibody, anti-LAMP1 antibody and DAPI respectively, and visualized by confocal microscopy (400X). b Transmission electron microscopy (TEM) analysis showing autophagosome (arrowed) after treated with Rapamycin (25 $\mu \mathrm{M})$, Ad-GFP, Ad-ATG5, Ad-ATG7 and Ad-ATG5 + Ad-ATG7 for $24 \mathrm{~h}$ in the C28l2 cells. The autophagosome is shown by red arrow. Rapamycin $(25 \mu \mathrm{M})$ used as a positive control, Bafilomycin A1 is a lysosomal inhibitor. c Qualitative analysis of LC3 and LAMP1 fluorescence intensity of chondrocytes under confocal microscopy. The values were normalized to the NC group. $\mathbf{d}$ Qualitative analysis of the number of autophagosome in chondrocytes under TEM. The values were normalized to the NC group 
ER stress-mediated cell apoptosis and cell cycle. As revealed in Fig. 5a and b, the expressions of cleaved caspase3 (16KD/18KD) and cleaved caspase12 (42KD) were markedly reduced in the $\mathrm{C} 28 \mathrm{I} 2$ cells infected with Ad-GFP, Ad-ATG5, Ad-ATG7 and Ad-ATG5 + Ad-ATG7. Rapamycin used as a positive control. It is suggested that not only Ad-ATG5 and Ad-ATG7 reduce apoptosis respectively, but also their combined effect can dramaticlly inhibit apoptosis compared with that of the control, the Ad-ATG5 and the Ad-ATG7 individually. Furthermore, FCM result also showed that the apoptosis rate markedly decreased in the C28I2 cells infected with Ad-ATG5 (6.35\%), Ad-ATG7 (9.00\%) and Ad-ATG5 + Ad-ATG7 (3.65\%) compared with that of the NC control (10.65\%) and the Ad-GFP control (14.1\%, Fig. 5c and d). The differences between each treatment group are of statistical significance $(P<0.05)$. The cell cycle distribution was analyzed by Flow cytometry analysis (FCM) as shown in Fig. 5e, $\mathrm{f}$ and $\mathrm{g}$. The data showed that the $\mathrm{S}$ phase proportion of C28I2 cells infected with Ad-ATG5 (58.12\%), Ad-ATG7 (55.63\%) and Ad-ATG5 +

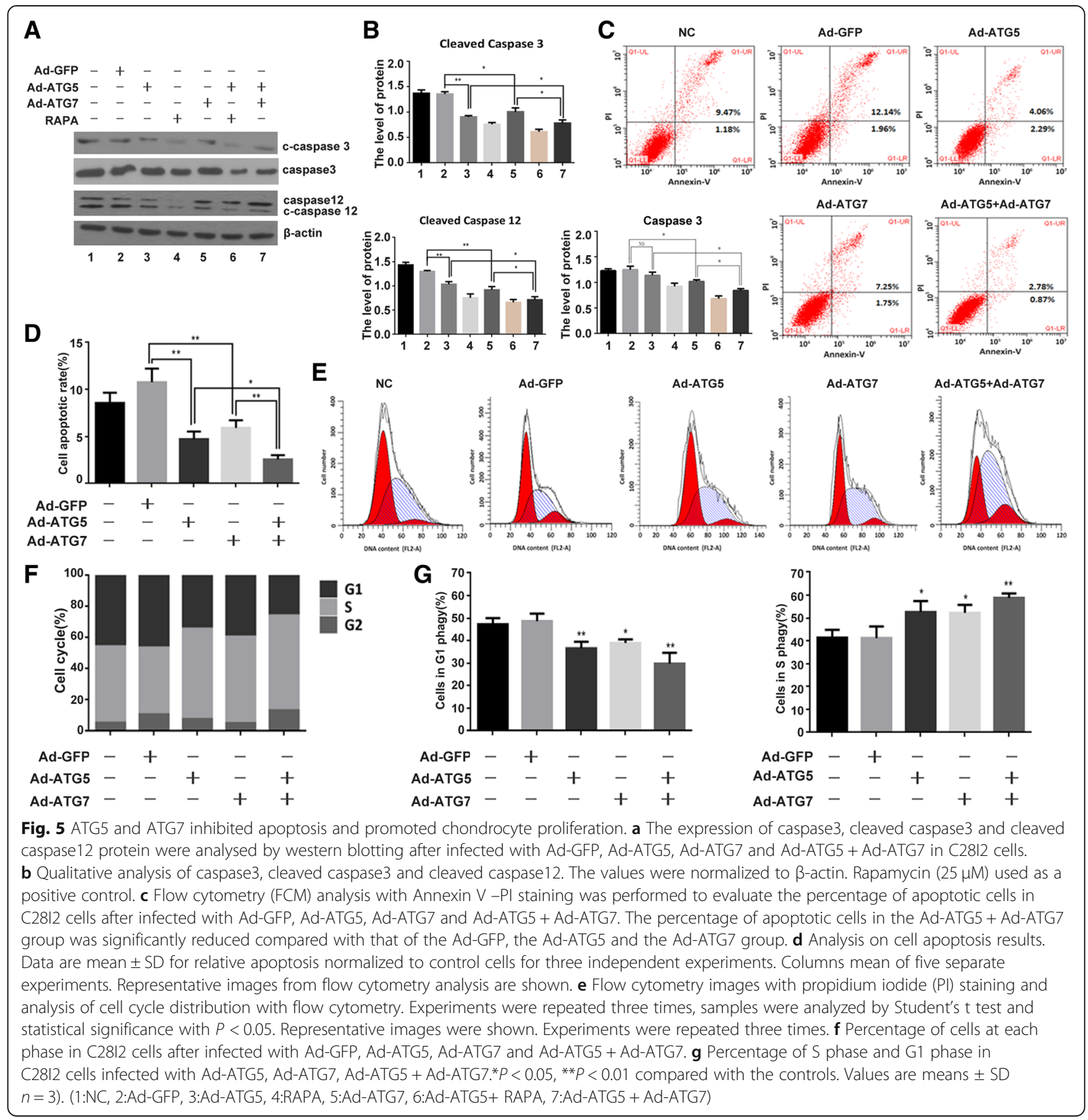


Ad-ATG7 (61.03\%) clearly increased compared with that of the Ad-GFP control (42.98\%). The G1 phase cell numbers were Ad-GFP (46.29\%), Ad-ATG5 (34.22\%), Ad-ATG7 (39.28\%) and Ad-ATG5 + Ad-ATG7 (25.71\%) respectively. It is suggested that the individual ATG5 or ATG7 can promote cells from G1 phase to $S$ phase and inhibit ER stress-mediated apoptosis in chondrocyte. Futhermore,their combined effect can significantly increase the cell number in $S$ phase and thus dramatically promote this cell proliferation enhancement and ER stress-mediated apoptosis repression.. The differences between treatment groups and control groups have statistical significance $(P<0.05)$.

\section{The effect of ATG5 and ATG7 on autophagy, ER stress, apoptosis and cell cycle through PERK signaling}

Autophagy as a cytoprotective mechanism to reduce the damaged organelles or degraded macromolecules in cells $[40,42]$. To further explore which UPR signal pathway is involved in autophagy and ER stress-mediated apoptosis, the expressions of PERK, p-PERK, Nrf2, IRE1, XBP1s/u, ATG5, LC3-I/LC3-II, P62 and ATG5-ATG12 were examined in the C28I2 cells infected with Ad-GFP, Ad-ATG5 + Ad-ATG7, Ad-ATG5 + Ad-ATG7 + Ad-siPERK, Ad-ATG5 + Ad-ATG7 + Ad-siNrf2, Ad-ATG5 + Ad-ATG7 + Ad-siPERK+Ad-siNrf2. As revealed in Fig. 6a and b, ATG5 + ATG7 increased autophagy and inhibited ER stress, however, the effect of ATG5 + ATG7 on ER stress repression was vanished in the $\mathrm{C} 28 \mathrm{I} 2$ cells treated with siPERK, siNrf2, siPERK+siNrf2,as revealed by enhanced expressions of PERK, p-PERK,Nrf2, IRE1, XBP1s/u.On the other side,the effect of ATG5 + ATG7 on autophagy enhancement was decreased in the C28I2 cells treated with siPERK, siNrf2, siPERK+siNrf2, as revealed by expressions of ATG5, LC3-I/LC3-II, P62 and ATG5-ATG12. Furthermore, after treated with siPERK, siNrf2,siPERK+siNrf2, the effect of ATG5 + ATG7 on apoptosis inhibition was eliminated, as shown by expressions of cleaved caspase 3 and cleaved caspase12 (Fig. 6c and d). FCM result also confirmed this result. The cell apoptotic rate was $4.17 \%$ in the C28I2 cells infected with Ad-ATG5 + Ad-ATG7, and increased in the C28I2 cells treated with Ad-ATG5 + Ad-ATG7 + siPERK (15.67\%), Ad-ATG5 + Ad-ATG7 + siNrf2 (24.41\%), Ad- ATG5 + Ad-ATG7 + siPERK+siNrf2 (27.02\%) (Fig. 6e and f). It is demonstrated that ATG5 and ATG7 increased autophagy, inhibit ER stress and apoptosis through PERK/Nrf2 signaling. Moreover, we detected cell cycle distribution of C28I2 cells in the presence of Ad-ATG5 + Ad-ATG7 + siPERK, Ad-ATG5 + Ad-ATG7 + siNrf2 and Ad-ATG5 + Ad-ATG7 + siPERK+siNrf2. The $\mathrm{S}$ phase cell number was $57.31 \%$ in the C28I2 cells treated with Ad-ATG5 + Ad-ATG7, and reduced in the C28I2 cells treated with
Ad-ATG5 + Ad-ATG7 + siPERK (51.22\%), Ad-ATG5 + Ad-ATG7 + siNrf2 (53.19\%), Ad-ATG5 + Ad-ATG7 + siPERK+siNrf2 (50.87\%), respectively. The data demonstrated that the effect of ATG5 + ATG7 on cell growth enhancement was decreased in the C28I2 cells treated with siPERK, siNrf2 and siPERK+siNrf2. The differences between the $\mathrm{S}$ phase cell number were up to statistical significance $(P<0.05)$ (Fig. $6 g$ and $h)$.

All is known that ATF4 is another downstream molecule of PERK, we also detected whether the effect of ATG5 and ATG7 is associated with ATF4. As revealed in Fig. 7a and b, the effect of ATG5 + ATG7 on ER stress repression was vanished in the siATF4-treated chondrocytes, however, this inhibition effect of siATF4 is blocked after siPERK treatment. On the other side, siATF4 decrease the effect of ATG5 + ATG7 on autophagy enhancement and apoptosis inhibition, and this effect of siATF4 is eliminated by siPERK (Fig. 7a and b).

Taken together, these results manifest the effect of ATG5 and ATG7 on autophagy, ER stress, cell cycle and apoptosis through PERK signalling. The protective effect of ATG5/7 overexpression on chondrocyte survival is dependent upon PERK signaling. Interestingly, the effect of siPERK and siNrf2 on the cytoprotective effect of ATG5/7 are of synergism, while the effect of siPERK and siATF4 are of antagonism. PERK signal may be the pivot for autophagy and ER stress.

\section{PERK is required for the effect of ATG5 and ATG7 on autophagy, ER stress, apoptosis and cell cycle}

We next used GSK2606414, a typical PERK pathway inhibitor, to validate the effect of ATG5 and ATG7 on autophagy and ER stress. As shown in Fig. 8a, b, c and d, ATG5 + ATG7 increased autophagy and inhibited ER stress, however, the effect of ATG5 + ATG7 on ER stress repression was vanished after treated with GSK2606414, as revealed by enhanced expressions of PERK, p-PERK, Nrf2, IRE1, XBP1s/u. On the other side, the effect of ATG5 + ATG7 on autophagy enhancement was reduced after treated with GSK2606414, as revealed by expressions of ATG5, LC3-I/LC3-II, P62 and ATG5-ATG12. Furthermore, after treated with GSK2606414, the effect of ATG5 + ATG7 on apoptosis inhibition was eliminated, as shown by enhanced expressions of cleaved caspase 3 and cleaved caspase12 (Fig. 8c and d). FCM result also proved this result. The cell apoptotic rate was $4.12 \%$ in the Ad-ATG5 + Ad-ATG7 infected C28I2 cells, and increased in the C28I2 cells treated with Ad-ATG5 + Ad-ATG7 + GSK2606414 (17.12\%) (Fig. 8e and f). The data demonstrated that ATG5 and ATG7 regulate autophagy, ER stress and apoptosis through PERK signaling.

Moreover, we detected cell cycle distribution of C28I2 cells in the presence of Ad-ATG5 + Ad-ATG7 and 


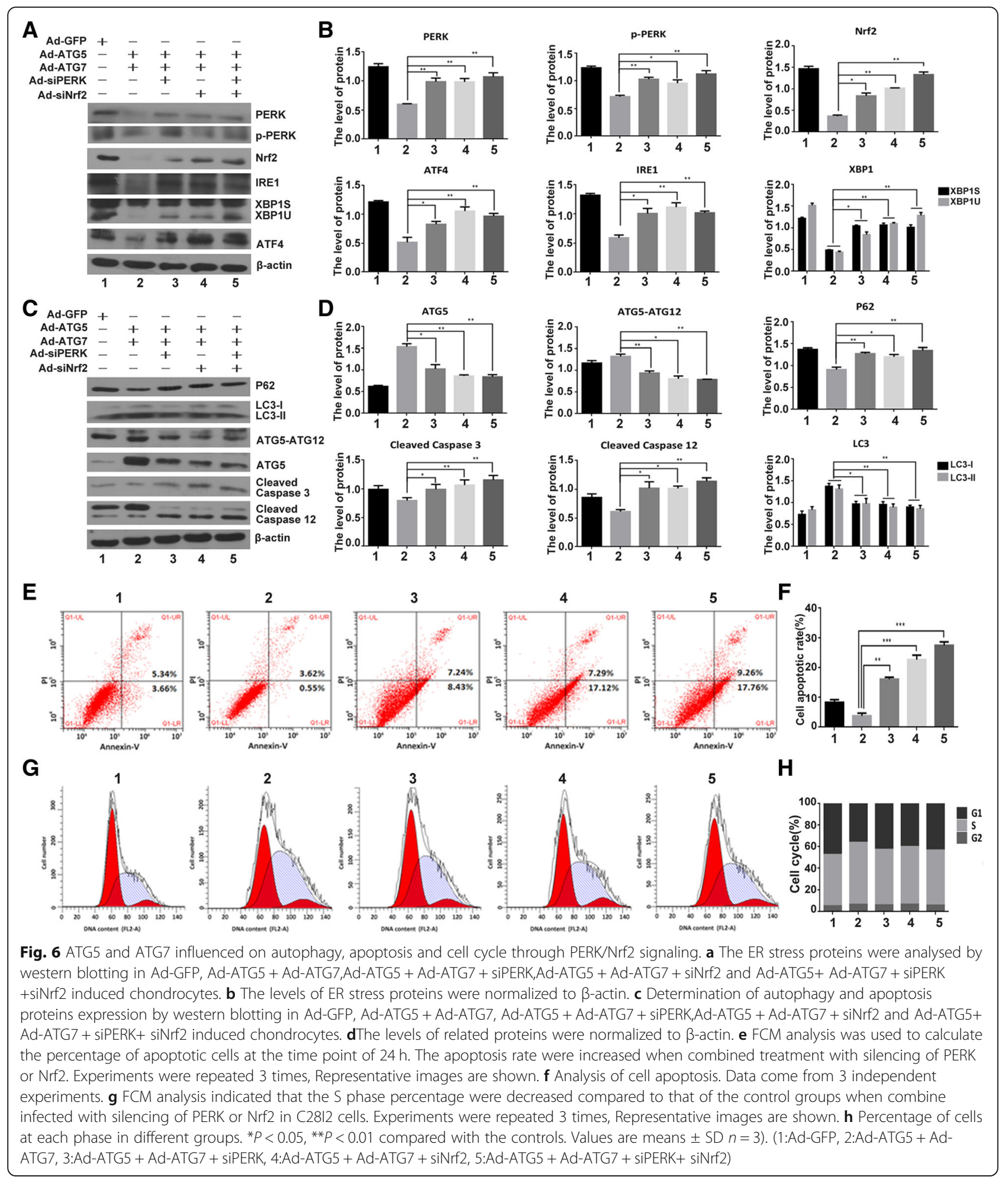

Ad-ATG5 + Ad-ATG7 + GSK2606414. The S phase cell number was $43.87 \%$ in the C28I2 cells treated with Ad-ATG5 + Ad-ATG7, and reduced in the C28I2 cells treated with Ad-ATG5 + Ad-ATG7 + GSK2606414 $(32.00 \%)$. The proportion of $\mathrm{S}$ phase were decreased after GSK2606414 treatment (Fig. 8g and h). It is showed that ATG5 and ATG7 promote cell proliferation through PERK signaling. The differences between the $S$ phase cell number were up to statistical significance $(P<0.05)$. Collectively, The effects of ATG5 

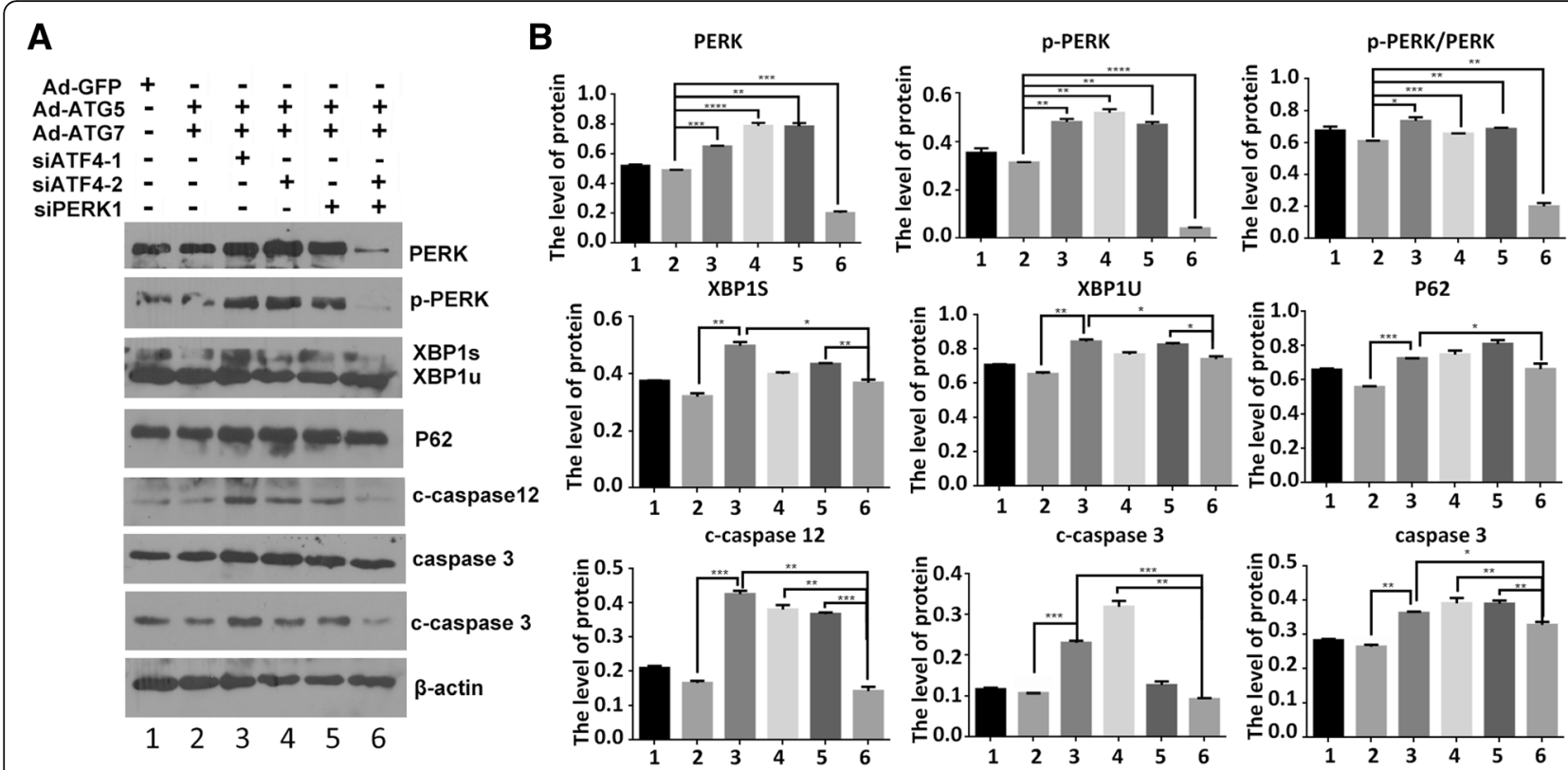

Fig. 7 ATG5 and ATG7 influenced on autophagy, apoptosis through PERK/ATF4 signaling. a The ER stress proteins were analysed by western blotting in Ad-GFP, Ad-ATG5 + Ad-ATG7,Ad-ATG5 + Ad-ATG7 + siATF4-1,Ad-ATG5 + Ad-ATG7 + siATF4-2,Ad-ATG5 + Ad-ATG7 + siPERK1 and AdATG5 + Ad-ATG7 + siPERK1+siATF4-2 induced chondrocytes. b The levels of related proteins were normalized to $\beta$-actin. (1:Ad-GFP, 2:Ad-ATG5 + Ad-ATG7, 3:Ad-ATG5 + Ad-ATG7 + siATF4-1, 4:Ad-ATG5 + Ad-ATG7+ siATF4-2, 5:Ad-ATG5 + Ad-ATG7 + siPERK1,

6:Ad-ATG5 + Ad-ATG7 + siPERK1+ SiATF4-2)

and ATG7 on autophagy, ER stress, apoptosis and cell cycle were through PERK signalling pathway.

\section{Discussion}

Cell survival, proliferation, autophagy and apoptosis are intimately connected processes, which are regulated by the mammalian target of rapamycin (mTOR) kinase and the ER stress pathway, also known as the UPR [43, 44]. As many literature reported, there are many connections between UPR and autophagy, such as, ATF6a branch which, as one of UPR pathway, is involved in the activation of mTORC1 and PERK which, as a major transducer of the ER stress, can mediate transcriptional activation of LC3 and ATG5 proteins in hypoxia. ATG5 and LC3 are involved in phagophore expansion and autophagosome formation [45-48]. Selective autophagy of the ER-ER-phagy, a particular type of autophagy, is involved in ER degradation and ER homeostasis. It is reported that different mammalian flavors of ER-phagy are mediated by different receptors. ER stress-induced receptor CCPG1 mediated ER-phagy requires ATG5, LC3 and FIP200. SEC62-mediated recovER-phagy requires ATG5, ATG7, and LC3. Most misfolded microbial-induced ER-phagy requires ATG7, ATG14, ATG16L1, BCN1 and FIP200. ER-phagy maintain the normal ER homeostasis and overall cell health through the degradation of ER membranes, removal of ER luminal protein aggregates $[49,50]$.
ATG7 is involved in two ubiquitin-like protein (Ubl) conjugation systems, the Atg12 conjugation system and the Atg8 conjugation system, as one of components during autophagy [51, 52]. Atg5 is critical for autophagy at the stage of autophagosome precursor synthesis. They participate in the initiation of autophagosome formation and ER-phagy process. However, whether and how their regulation of autophagy is associated with ER stress and apoptosis remained unknown. This study can clarify the relationship between autophagy, ER stress and apoptosis, as well as the molecular events in this ER-phagy process, which have important pathophysiological consequences.

Many studies have shown that both autophagy and UPR are activated under ER stress, and promote cell survival. As the UPR induces not only cell survival but also cell death signals, the well-orchestrated processes between autophagy and UPR are involved in either restoring cellular homeostasis or committing to cell death [53, 54]. We detected the expressions of autophagy related proteins increased in Tm-induced ER stress, and the expression of ER stress associated proteins, PERK、 p-PERK and Nrf2, decreased dramatically in RAPA-stimulated autophagy. Interestingly, we found the expression of autophagy proteins were partially inhibited after silencing of PERK, Nrf2 or ATF4 via siRNA approach. These results suggest that ER stress interplays with autophagy in human chondrocyte (Fig. 1). 
Autophagy, as a well-defined and self-digestion process for degrading proteins and organelles in response to cellular stress, can maintain the cell's homeostasis and conducive to cell survival. More than 30 autophagy-related (ATG) genes control autophagy activation. ATG5 and ATG7, as autophagy proteins, can participate in the autophagosome formation [55, 56]. To define the interaction between autophagy, ER stress, and apoptosis, we generated adenovirus carrying ATG5 and ATG7, then infected the C28I2 cells. The result showed that overexpression of ATG5 or ATG7 can improve autophagy, increase autophagy

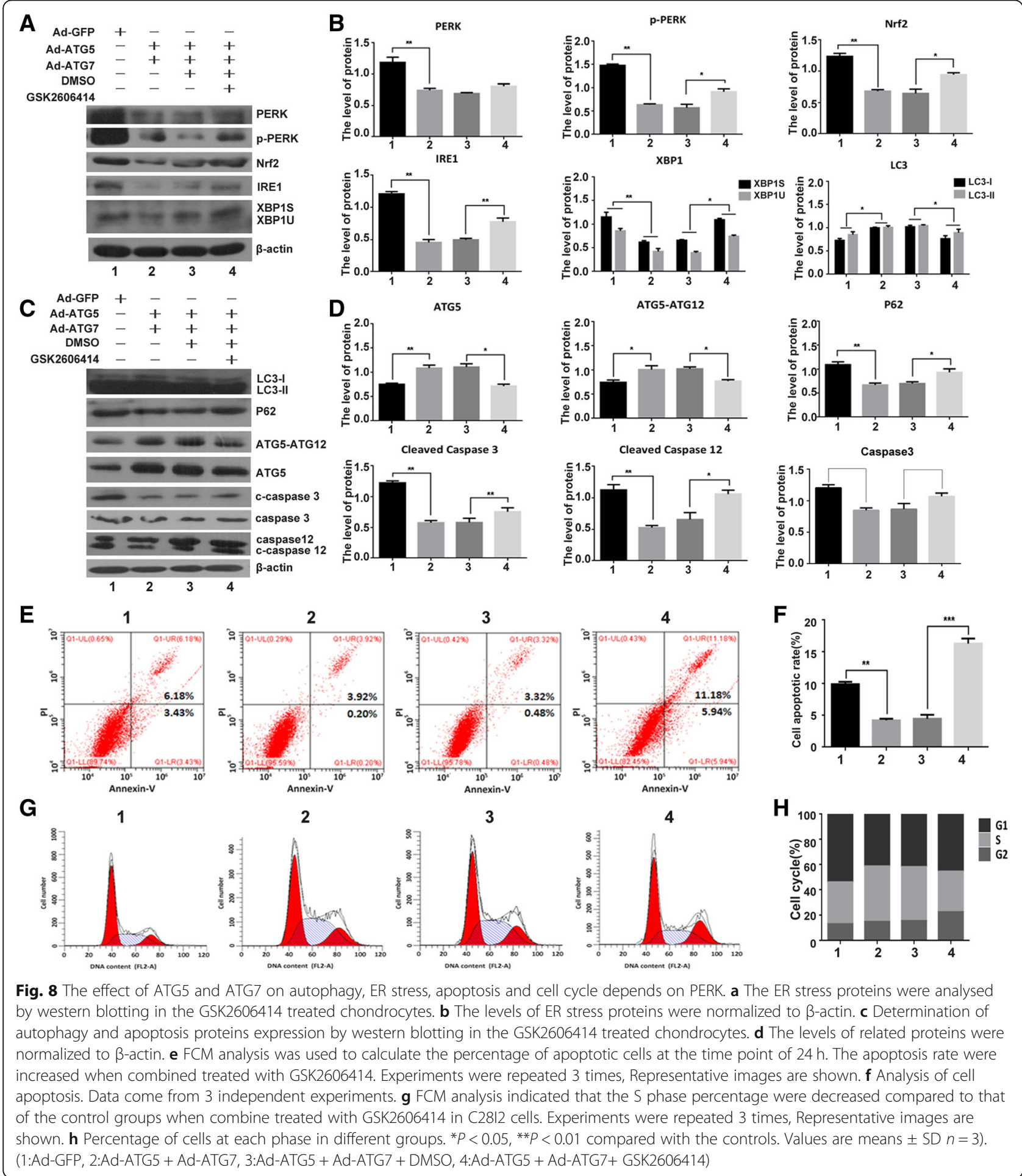


flux and autophosome formation. Meanwhile, the ER stress was inhibited after above mentioned cells being infected with Ad-ATG5 or Ad-ATG7,as assayed by PERK, p-PERK and Nrf2 expression. Additionally, the combined effect of ATG5 and ATG7 can evidently enhance autophagy and inhibit ER stress comparing with individual treatment (Figs. 2, 3, 4).

Autophagy and apoptosis determine a cell's fate through regulating the turnover of proteins and organelles. Normally cytoprotective function of autophagy inhibits the apoptosis induction, and serves to cell survival through suppressing apoptosis. Most of apoptosis-associated caspase activation shuts off the autophagic process and block autophagy. ATG7, an E1-like enzyme, is required to inhibit translocation of caspase- 9 to the apoptosome, hence preventing apoptosis $[57,58]$. ATG5 can also participate in the protective effect. The level of active caspase-3, 7 and RAPA degradation in CsA-treated cells increased after autophagy effectors ULK1, ATG5 or ATG7 were silenced [59-61].

Our data indicated that overexpression of ATG5, ATG7 and ATG5 + ATG7 can clearly reduce the apoptosis of chondrocyte comparing with the control's. And the joint infection of them can enhance the inhibition effect. Moreover, overexpression of them can also influence cell cycle distribution. The application of ATG5, ATG7 and ATG5 + ATG7 can promote cell proliferation in chondrocyte with the $\mathrm{G1}$ phase cells reduction, and the $S$ phase cells enhancement. The joint application of ATG5 and ATG7 can promote the increasing effect of the $\mathrm{S}$ phase cells. These data showed that overexpression of ATG5, ATG7 and ATG5 + ATG7 can obviously inhibit apoptosis and improve cell proliferation in chondrocytes (Figs. 5).
All is known that chondrocyte apoptosis is the main cause of osteoarthritis. When cartilage destruction and matrix degradation failed to be eliminated and remain in cartilage, they can induce the chondrocyte apoptosis. Autophagy induction might be a beneficial method to relieve chondrocyte apoptosis and protect cartilage from destruction [62, 63]. Herein, we tested that overexpression of ATG5 and ATG7 can promote cell proliferation and inhibit apoptosis when they activate autophagy. There have been recent reviews on the bidirectional interaction between autophagy and ER stress. The degree of interaction between autophagy and UPR activation can determine the balance between prosurvival and antisurvival signals, in which autophagy is involved in either promote or attenuate ERS and UPR signals. We detected that both ATG5 and ATG7 overexpression can block UPR as soon as autophagy activation. It is demonstrated that both ATG5 and ATG7 can stimulate autophagy, which leads to protective effect and serves as a prosurvival mechanism through inhibition ER stress conditions (Fig. 6a, b, c, d).

Eukaryotic cell growth and proliferation are controlled by cell cycle, in which cell cycle arrest is often accompanied by autophagy induction, a crucial survival procedure during stress conditions. The above studies suggest that ATG5 and ATG7 increase the cell proliferation and inhibit ER stress and apoptosis in chondrocyte, however, this enhancement effect of ATG5 and ATG7 vanished after the cells being infected with Ad-siPERK, Ad-siNrf2 and Ad-siPERK+Ad-siNrf2 as evidenced by G1 phase arresting, $S$ phase reducing and G2-M phase delaying (Fig. 6e, f, g, h). More importantly, the protective effect of ATG5/7 overexpression on chondrocyte survival is dependent upon PERK signaling. Interestingly,

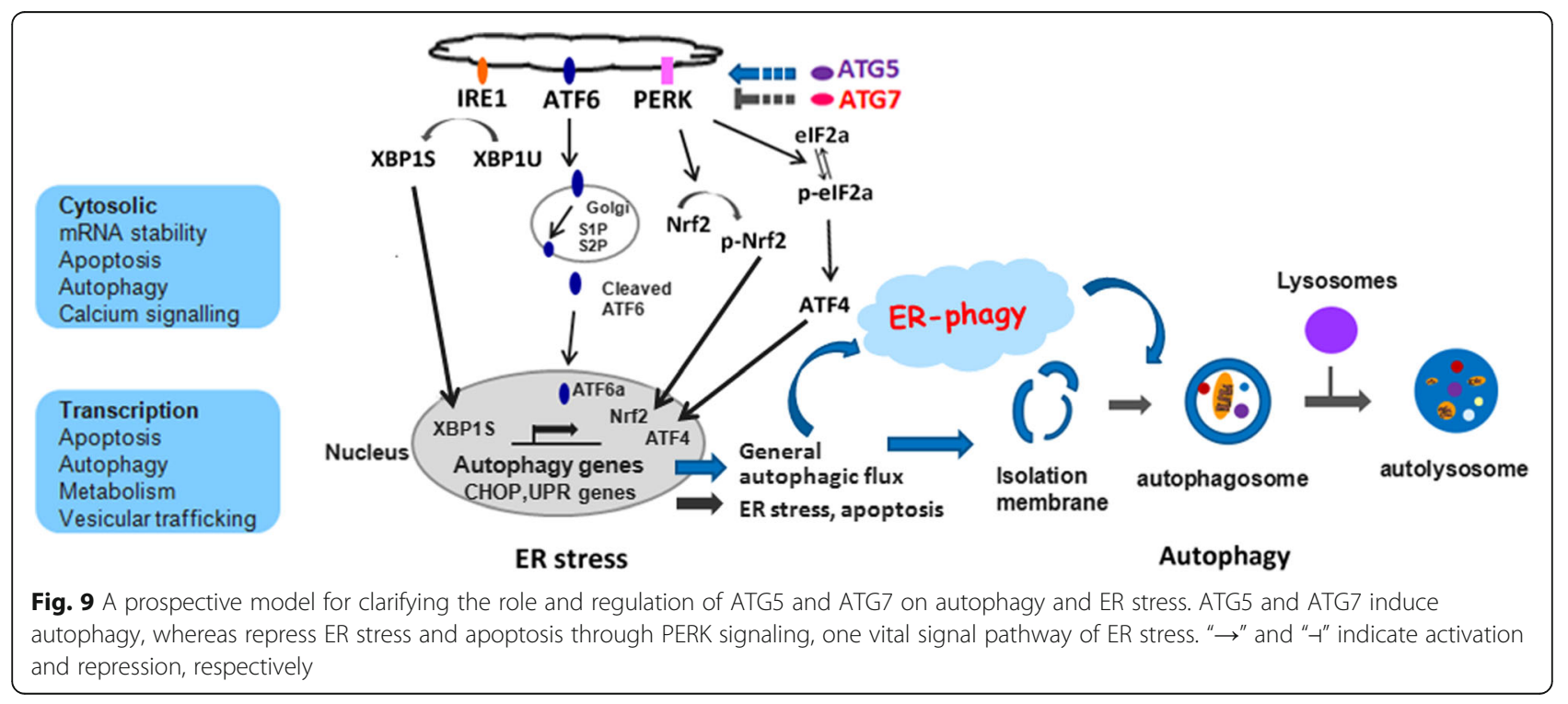


the effect of siPERK and siNrf2 on the cytoprotective effect of ATG5/7 are of synergism, while the effect of siPERK and siATF4 are of antagonism (Fig. 7). The effects of ATG5 + ATG7 on ER stress repression, autophagy enhancement, apoptosis inhibition and cell growth augment declined after treatment with siPERK and GSK2606414, a typical PERK pathway inhibitor (Fig. 8). It is indicated that ATG5 and ATG7 regulate on autophagy, ER stress, apoptosis and cell cycle through PERK signalling pathway, a vital branch UPR pathways. As reported, ATG5 and ATG7 are involved in autophosome formation and fusion of autophagosomes and lysosomes subsequently through ER-phagy, which contains a variety of processes that are both mechanistically different and regulate the delivery of ER fragments or their luminal content within lysosomes. The ER-phagy process modulates ER turnover, ER size, and clearance of ER subdomains containing proteins and lipids that are faulty or present in excess. PERK was reported to up-regulate the transcription of numerous autophagy genes and cargo receptors through ATF4 and CHOP, resulting in an augment in autophagic flux [50, 64, 65]. Our results showed that the protective effect of ATG5 and ATG7 overexpression on chondrocyte survival is dependent upon PERK signaling through ER-phagy process, suggesting that PERK signal is the pivot role for ER-phagy in normal ER homeostasis and overall cell health.

More recently, autophagy can be regulated by cell cycle control, and has been shown to engage in a complex interplay with ER stress and apoptosis. In cellular procedure, autophagy, ER-phagy and UPR act as a cell survival pathway to suppress apoptosis, and on the other hand, it can result in cell death.The molecular regulators of every pathway are interconnected and affect each other. The cross-talk among autophagy, ER stress, apoptosis and cell growth are quite intricate. All of them regulate the overall fate of the cell synergistically.

\section{Conclusions}

Our study provides a novel insight into the role of ATG5 and ATG7 in regulating autophagy, ER stress, ER-phagy, apoptosis and cell proliferation. As summarized in Fig. 9, we propose a model for the different role of ATG5 and ATG7 in autophagy and ER stress. ATG5 and ATG7 induce autophagy, autophagy flux and autophagosome formation, whereas inhibit ER stress in the process of cell survival and cell death through PERK signaling, one vital signal pathway of ER stress. Continued research of these and other means of crosstalk between ER stress, apoptosis and autophagy is necessary to elucidate the mechanisms controlling the balance between survival and death both under normal and disease conditions. New insight into the mechanism of autophagy affect ER stress responses will open new approach to the involvement of ER-phagy in ER homeostasis and the development of molecular target-based treatment of cartilage disorders and osteoarthritis.

\section{Additional file}

Additional file 1: The results of DNA sequencing and the adenoviral vector endonuclease identification. (PDF $344 \mathrm{~kb}$ )

\section{Abbreviations}

ATF6: activating transcription factor 6; ER: endoplasmic reticulum; IRE1: inositol-requiring enzyme 1; LAMP-1: lysosome-associated membrane proteins 1; LC3: Microtubule-associated protein 1a/ $\beta$-light chain 3; PERK: protein kinase RNA (PKR)-like ER kinase; PI: propidium iodide; TEM: transmission electron microscope; Tg: thapsigargin; Tm: tunicamycin; UPR: unfolded protein response

\section{Acknowledgements}

This work was aided by the National Science Foundation of China (No.81672209, No.81871769).

\section{Availability of data and materials}

The datasets used and/or analysed during the current study are available from the corresponding author on reasonable request.

\section{Authors' contributions}

We are committed to study ER stress, autophagy and their relation with bone development and bone associated diseases. All authors participated in the design, interpretation of the studies and analysis of the data and review of the manuscript; and all authors approved the final version to be published. ZW, XWW, YDY, LR, LM were master students. They conducted the experiments; ZW, XWW and YDY analysed and interpretated the data; Prof. Guo Fj designed the manuscript and had full access to all of the data in the study and takes responsibility for the integrity of the data and the accuracy of the data analysis. All authors have read and approved the manuscript prior to submission.

Ethics approval and consent to participate Not applicable.

Consent for publication

Not applicable.

Competing interests

The authors declare that they have no competing interest.

\section{Publisher's Note}

Springer Nature remains neutral with regard to jurisdictional claims in published maps and institutional affiliations.

Received: 30 January 2019 Accepted: 13 April 2019

Published online: 06 May 2019

\section{References}

1. Amber NH, Dan JL, Thomas RG, Edmund BR. Autophagy: regulation and role in development. Autophagy. 2013;9(7):951-72.

2. Chun Y, Kim J. Autophagy: an essential degradation program for cellular homeostasis and life. Cells. 2018;7(12):278.

3. White E, Mehnert JM, Chan CS. Autophagy, metabolism, and Cancer. Clin Cancer Res. 2015;21(22):5037-46.

4. Mathiassen SG, De Zio D, Cecconi F. Autophagy and the cell cycle: a complex landscape. Front Oncol. 2017;7:51.

5. Rubinsztein DC, Gestwicki JE, Murphy LO, Klionsky DJ. Potential therapeutic applications of autophagy. Nat Rev Drug Discov. 2007;6:304-12. 
6. Romanov J, Walczak M, Ibiricu I. Mechanism and functions of membrane binding by the Atg5-Atg12/Atg16 complex during autophagosome formation. EMBO J. 2012;31(22):4304-17.

7. $\mathrm{M} \mathrm{W}$, Martens S. Dissecting the role of the Atg12-Atg5-Atg16 complex during autophagosome formation. Autophagy. 2013;9(3):424-5.

8. Matsushita M, Suzuki NN, Obara K, Fujioka Y, Ohsumi Y, Inagaki F. Structure of Atg5.Atg16, a complex essential for autophagy. J Biol Chem. 2007;282:6763-72

9. Yang Z, Klionsky DJ. Mammalian autophagy: core molecular machinery and signaling regulation. Curr Opin Cell Biol. 2010;22:124-31.

10. Kraft C, Martens S. Mechanisms and regulation of autophagosome formation. Curr Opin Cell Biol. 2012;24:496-501.

11. Nakatogawa $H$, Ichimura $Y$, Ohsumi $Y$. Atg8, a ubiquitin-like protein required for autophagosome formation, mediates membrane tethering and hemifusion. Cell. 2007:130:165-78.

12. Fribley A, Zhang K, Kaufman RJ. Regulation of apoptosis by the unfolded protein response. Methods Mol Biol. 2009;559:191-204.

13. Pluquet $\mathrm{O}$, Pourtier $\mathrm{A}$, Abbadie $\mathrm{C}$. The unfolded protein response and cellular senescence. A review in the theme: cellular mechanisms of endoplasmic reticulum stress signaling in health and disease. Am J Physiol Cell Physiol. 2015;308:C415-25.

14. Grootjans J, Kaser A, Kaufman RJ, Blumberg RS. The unfolded protein response in immunity and inflammation. Nat Rev Immunol. 2016:16:469-84.

15. Lamb CA, Yoshimori T, Tooze SA. The autophagosome: origins unknown, biogenesis complex. Nat Rev Mol Cell Biol. 2013;14:759-74.

16. Lahiri $\mathrm{V}$, Klionsky DJ. CCPG1 is a noncanonical autophagy cargo receptor essential for reticulophagy and pancreatic ER proteostasis. Autophagy. 2018;14(7):1107-9.

17. Mizushima N. A dual binding receptor for ER-phagy. Dev Cell. 2018:44(2):133-5

18. Horiuchi $\mathrm{K}$, Tohmonda T, Morioka H. The unfolded protein response in skeletal development and homeostasis. Cell Mol Life Sci. 2016;73:2851-69.

19. Zuscik MJ, Hilton MJ, Zhang X, Chen D, O'Keefe RJ. Regulation of chondrogenesis and chondrocyte differentiation by stress. J Clin Invest. 2008;118:429-38.

20. Saito A, Imaizumi K. Endoplasmic reticulum stress response in osteogenesis. Clin Calcium. 2013;23:1569-75.

21. Walter F, Schmid J, Düssmann H, Concannon CG, Prehn JH. Imaging of single cell responses to ER stress indicates that the relative dynamics of IRE1/XBP1 and PERK/ATF4 signalling rather than a switch between signalling branches determine cell survival. Cell Death Differ 2015;22:1502-16.

22. Teske BF, Wek SA, Bunpo P, Cundiff JK, McClintick JN, Anthony TG, Wek RC. The eIF2 kinase PERK and the integrated stress response facilitate activation of ATF6 during endoplasmic reticulum stress. Mol Biol Cell. 2011;22(22):4390-405.

23. Han X, Zhang P, Jiang R, Xia F, Li M, Guo FJ. Explore on the effect of ATF6 on cell growth and apoptosis in cartilage development. Histochem Cell Biol. 2014;142(5):497-509.

24. Appenzeller-Herzog C, Hall MN. Bidirectional crosstalk between endoplasmic reticulum stress and mTOR signaling. Trends Cell Biol. 2012;22(5):274-82.

25. Ogata M, Hino S, Saito A, Morikawa K, Kondo S, Kanemoto S, et al. Autophagy is activated for cell survival after endoplasmic reticulum stress. Mol Cell Biol. 2006;26:9220-31.

26. Jia W, Pua HH, Li QJ, He YW. Autophagy regulates endoplasmic reticulum homeostasis and calcium mobilization in T lymphocytes. J Immunol. 2011;186:1564-74.

27. Chen X, Li M, Chen D, Gao W, Guan JL, Komatsu M, Yin XM Autophagy induced by calcium phosphate precipitates involves endoplasmic reticulum membranes in autophagosome biogenesis. PLoS One. 2012;7:e52347.

28. Bouderlique T, Vuppalapati KK, Newton PT, et al. Targeted deletion of Atg5 in chondrocytes promotes age-related osteoarthritis. Ann Rheum Dis. 2016;75(3):627-31.

29. Carames B, Taniguchi N, Otsuki S, Blanco FJ, Lotz M. Autophagy is a protective mechanism in Normal cartilage and its aging-related loss is linked with cell death and osteoarthritis. Arthritis Rheum. 2010:62(3):791-801.

30. Carames B, Hasegawa A, Taniguchi N, Miyaki S, Blanco FJ, Lotz M. Autophagy Activation by Rapamycin Reduces Severity of Experimenta Osteoarthritis. Ann Rheum Dis. 2012;71(4):575-81.
31. Miyake S, Makimura M, Kanegae Y, Harada S, Sato Y, Takamori K, Tokuda C, Saito I. Efficient generation of recombinant adenoviruses using adenovirus DNA-terminal protein complex and a cosmid bearing the full-length virus genome. Proc Natl Acad Sci U S A. 1996:93:1320-4.

32. Wu ZM, Li ML, Zheng W, Hu Q, Cheng Z, Guo FJ. Silencing of both ATF4 and PERK inhibits cell cycle progression and promotes the apoptosis of differentiating chondrocytes. Int J Mol Med. 2017;40:101-11.

33. Brown M, Wittwer C. Flow cytometry: principles and clinical applications in hematology. Clin Chem. 2000;46(8 Pt 2):1221-9.

34. Radcliff $\mathrm{G}$, Jaroszeski MJ. Basics of flow cytometry. Methods Mol Biol. 1998:91:1-24.

35. Guo FJ, Liu Y, Zhou J, Luo S, Zhao W, Li X, Liu C. XBP1S protects cells from ER stress-induced apoptosis through Erk1/2 signaling pathway involving CHOP. Histochem Cell Biol. 2012;138:447-60.

36. Guo F, Jiang R, Xiong Z, Xia F, Li M, Chen L, Liu CJ.IRE1a constitutes a negative feedback loop with BMP2 and acts as a novel mediator in modulating osteogenic differentiation. Cell Death Dis. 2014;22:5:e1239.

37. Zhou C, Zhong W, Zhou J, Sheng F, Fang Z, Wei Y, Chen Y, Deng X, Xia B, Lin J. Monitoring autophagic flux by an improved tandem fluorescenttagged LC3 (mTagRFP-mWasabi-LC3) reveals that high-dose rapamycin impairs autophagic flux in cancer cells. Autophagy. 2012;8(8):1215-26.

38. Bampton ET, Goemans CG, Niranjan D, Mizushima N, Tolkovsky AM. The dynamics of autophagy visualized in live cells: from autophagosome formation to fusion with endo/lysosomes. Autophagy. 2005;1:23-36.

39. Du Toit A, Hofmeyr JS, Gniadek TJ, Loos B. Measuring autophagosome flux. Autophagy. 2018;14(6):1060-71.

40. Huynh KK, Eskelinen E-L, Scott CC, Malevanets A, Saftig P, Grinstein S. LAMP proteins are required for fusion of lysosomes with phagosomes. EMBO J. 2007;26:313-24.

41. King BC, Renström E, Blom AM.Intracellular cytosolic complement component C3 regulates cytoprotective autophagy in pancreatic beta cells by interaction with ATG16L1.Autophagy. 2019;15(5):919-921.

42. Tang Z, Takahashi Y, Chen C, Liu Y, He H, Tsotakos N, Serfass JM, Gebru MT, Chen $\mathrm{H}$, Young MM, Wang HG. Atg2A/B deficiency switches cytoprotective autophagy to non-canonical caspase-8 activation and apoptosis. Cell Death Differ. 2017;24(12):2127-2138.

43. Ciechomska IA, Gabrusiewicz K, Szczepankiewicz AA, Kaminska B. Endoplasmic reticulum stress triggers autophagy in malignant glioma cells undergoing cyclosporine A-induced cell death. Oncogene. 2013;32:1518-29.

44. Jager R, Bertrand MJM, Gorman AM, Vandenabeele P, Samali A. The unfolded protein response at the crossroads of cellular life and death during endoplasmic reticulum stress. Biol Cell. 2012;104:259-70.

45. Jung $\mathrm{CH}$, Ro S-H, Cao J, Otto NM, Kim D-H. mTOR regulation of autophagy. FEBS Lett. 2010;584:1287-95

46. Kroemer G, Mariño G, Levine B. Autophagy and the integrated stress response. Mol Cell. 2010;40(2):280-93.

47. Kato H, Nakajima S, Saito Y, Takahashi S, Katoh R, Kitamura M. mTORC serves ER stress-triggered apoptosis via selective activation of the IRE1-JNK pathway. Cell Death Differ. 2012;19(2):310-20.

48. Reiling JH, Sabatini DM. Increased mTORC1 signaling UPRegulates stress. Mol Cell. 2008:29(5):533-5.

49. Smith MD, Harley ME, Kemp AJ, Wills J, Lee M, Arends M, von Kriegsheim A, Behrends C, Wilkinson S. CCPG1 is a non-canonical autophagy cargo receptor essential for ER-Phagy and pancreatic ER Proteostasis. Dev Cell. 2018;44(2):217-232.e11.

50. Smith M, Wilkinson S. ER homeostasis and autophagy. Essays Biochem. 2017:61:625-35

51. Mizushima N, Yoshimori T, Ohsumi Y. The role of Atg proteins in autophagosome formation. Annu Rev Cell Dev Biol. 2011;27:107-32.

52. Xie Z, Klionsky DJ. Autophagosome formation: core machinery and adaptations. Nat Cell Biol. 2007;9(10):1102-9.

53. Høyer-Hansen $M$, Jäättelä M. Connecting endoplasmic reticulum stress to autophagy by unfolded protein response and calcium. Cell Death Differ. 2007;14(9):1576-82

54. Song S, Tan J, Miao Y, Li M, Zhang Q. Crosstalk of autophagy and apoptosis: involvement of the dual role of autophagy under ER stress. J Cell Physiol. 2017;232(11):2977-84.

55. Codogno P, Meijer AJ. Autophagy and signaling: their role in cell survival and cell death. Cell Death Differ. 2005;12(Suppl 2):1509-18.

56. Mizushima N, Levine B, Cuervo AM, Klionsky DJ. Autophagy fights disease through cellular self-digestion. Nature. 2008;451:1069-75. 
57. Li M, Gao P, Zhang J. Crosstalk between autophagy and apoptosis: potential and emerging therapeutic targets for cardiac diseases. Int J Mol Sci. 2016;17:1-19.

58. Han J, Hou W, Goldstein LA, Stolz DB, Watkins SC, Rabinowich H. A complex between Atg7 and Caspase-9: a novel mechanism of cross-regulation between autophagy and apoptosis. J Biol Chem. 2014;289:6485-97.

59. Rouschop KM, van den Beucken T, Dubois L, Niessen H, Bussink J, Savelkouls K, et al. The unfolded protein response protects human tumor cells during hypoxia through regulation of the autophagy genes MAP1LC3B and ATG5. J Clin Invest. 2010;120:127-41.

60. Ciechomska IA, Kaminska B. ER stress and autophagy contribute to CsAinduced death of malignant glioma cells. Autophagy. 2012;8(10):1526-8.

61. Kim KH, Lee MS. Autophagy — a key player in cellular and body metabolism. Nat Rev Endocrinol. 2014;10(6):322-37.

62. Hyun SH, Hyun AK. Chondrocyte apoptosis in the pathogenesis of osteoarthritis. Int J Mol Sci. 2015;16(11):26035-54.

63. Figueroa PL, Lotz M, Blanco FJ, Carames B. Autophagy Activation Protects from Mitochondrial Dysfunction in Human Chondrocytes. Arthritis Rheumatol. 2015;67(4):966-76.

64. Clarke AJ, Simon AK. Autophagy in the renewal, differentiation and homeostasis of immune cells. Nat Rev Immunol. 2019;19:170-83.

65. Grumati P, Dikic I, Stolz A. ER-phagy at a glance. J Cell Sci. 2018;131:1-6. jcs217364. https://doi.org/10.1242/jcs.217364.

Ready to submit your research? Choose BMC and benefit from:

- fast, convenient online submission

- thorough peer review by experienced researchers in your field

- rapid publication on acceptance

- support for research data, including large and complex data types

- gold Open Access which fosters wider collaboration and increased citations

- maximum visibility for your research: over $100 \mathrm{M}$ website views per year

At $\mathrm{BMC}$, research is always in progress.

Learn more biomedcentral.com/submissions 